\title{
INFLUÊNCIA DO TIPO DE TRANSDUTOR \\ NO MASCARAMENTO EM DEFICIÊNCIA \\ AUDITIVA CONDUTIVA E MISTA BILATERAL
}

\section{Influence of transducer's type in bilateral conductive \\ and mixed hearing loss masking}

Lívia Tamie Oshiro (1), Marcela Rosana Maia da Silveira ${ }^{(2)}$, Daniela Gil (3)

\begin{abstract}
RESUMO
Objetivo: verificar a influência do tipo de transdutor na quantidade de mascaramento necessária para obtenção de limiares por vias aérea e óssea em indivíduos com deficiência auditiva condutiva e mista. Método: foram avaliados 16 indivíduos com presença de deficiência auditiva condutiva ou mista bilateral simétrica com gap aéreo-ósseo de no mínimo 15dBNA. Os indivíduos foram submetidos à anamnese, meatoscopia, medidas de imitância acústica, audiometria tonal liminar e audiometria vocal, realizadas com fones supra-aurais TDH-39 e com os fones de inserção ER-3A. Resultados: não houve diferença estatisticamente significante entre as orelhas independentemente do tipo de transdutor. A quantidade de mascaramento utilizada com o fone de inserção ER-3A para testar a via aérea, nas frequências de $250 \mathrm{~Hz}$ e $500 \mathrm{~Hz}$, foi menor do que a utilizada com o fone supra-aural, com significância estatística. Conclusões: há influência do tipo de transdutor na quantidade de mascaramento utilizada para obtenção dos limiares de via aérea e via óssea, principalmente nas frequências baixas, sendo que a quantidade de mascaramento com o fone de inserção é menor tanto para testar a via aérea como a via óssea.
\end{abstract}

DESCRITORES: Perda Auditiva Condutiva; Mascaramento Perceptivo; Auxiliares de Audição; Audiometria de Tons Puros

\section{INTRODUÇÃO}

Para a obtenção dos limiares auditivos tanto para tons puros quanto para tons de fala, existem três tipos de fones auriculares: os fones supraaurais, circum-aurais e de inserção. Os fones supra-aurais são os transdutores convencionais em audiologia clínica, nos quais o coxim do fone é

(1) Fonoaudióloga graduada na Universidade Federal de São Paulo, UNIFESP, São Paulo, SP, Brasil.

(2) Fonoaudióloga pós graduanda, nível mestrado do Programa em Distúrbio da Comunicação Humana da Universidade Federal de São Paulo, UNIFESP, São Paulo, SP, Brasil.

(3) Fonoaudióloga; Professora Doutora adjunto da Disciplina dos Distúrbios da Audição do Departamento de Fonoaudiologia da Universidade Federal de São Paulo, UNIFESP, São Paulo, SP, Brasil; Doutora em Ciências pela Universidade Federal de São Paulo.

Conflito de interesses: inexistente pressionado contra o pavilhão auricular. Nos fones circum-aurais, o coxim do fone fica posicionado ao redor do pavilhão auricular ao invés de pressionálo. Já os fones de inserção, são adaptados dentro do meato acústico externo por meio de plugs de espuma descartáveis, reduzindo a área do crânio exposta ao estímulo sonoro. Os fones de inserção foram desenvolvidos para imitar a resposta por frequências dos fones supra-aurais TDH-39, entretanto, para que se correlacione os limiares auditivos tonais obtidos com fones supra-aurais e fones de inserção em equipamentos calibrados para os fones convencionais, é necessário utilizar fatores de correção ${ }^{1}$.

Os fones de inserção promovem maior conforto para o paciente; reduzem a ocorrência de colabamento do meato acústico externo; atenuam o ruído ambiental, aumentam a atenuação interaural tanto para os tons puros quanto para a fala, reduzindo ou 
até eliminando a necessidade de mascaramento; aumentam a confiabilidade do teste-reteste; são mais higiênicos, já que os plugs são descartáveis; promovem uma conversão precisa dos limiares auditivos para nível de pressão sonora do ganho e da saturação das próteses auditivas entre outras ${ }^{2,3}$.

Estudos apontam que os valores de atenuação interaural obtidos com os fones de inserção são maiores quando comparados com os obtidos com os fones supra-aurais na faixa de 250 a $8000 \mathrm{~Hz}^{1,4-10}$.

A avaliação audiológica de indivíduos com deficiência auditiva condutiva e/ou mista bilateral é encarada como um dilema em audiologia clínica, no que diz respeito ao uso do mascaramento, pois devido ao rebaixamento da via aérea e preservação da via óssea, muitas vezes o nível de mascaramento não é suficiente para eliminar a participação da orelha não testada ou excede o valor de atenuação interaural, gerando a ocorrência do supermascaramento e consequentemente imprecisão quanto aos limiares obtidos ${ }^{11,12}$.
Com base nestas evidências, o objetivo deste estudo foi verificar a influência do tipo de transdutor na quantidade de mascaramento necessária para obtenção de limiares auditivos por via aérea e via óssea em indivíduos com deficiência auditiva condutiva e mista bilateral.

\section{MÉTODO}

A seleção da amostra obedeceu aos seguintes critérios de inclusão: idade entre 15 e 70 anos; média dos limiares de 500, 1000 e $2000 \mathrm{~Hz}$ até 55 $\mathrm{dB}$; simetria entre os limiares auditivos das duas orelhas; apresentar gap aéreo-ósseo de no mínimo $15 \mathrm{~dB}$ e no máximo 60 dB entre 500 e 4000 Hz; não apresentar síndromes ou histórico de comprometimentos cognitivos.Todos os selecionados foram atendidos nos Ambulatórios de Audiologia Clínica, da Disciplina de Distúrbios da Audição da Universidade Federal de São Paulo (UNIFESP).As características da amostra estão apresentadas na Figura 1.

\begin{tabular}{|c|c|c|c|c|}
\hline Indivíduos & Sexo & Idade & Tipo de DA & Grau de DA \\
\hline 1 & Masculino & 28 anos & Condutivo & Leve \\
\hline 2 & Feminino & 16 anos & Condutivo & Leve \\
\hline 3 & Feminino & 24 anos & Condutivo & Leve \\
\hline 4 & Feminino & 70 anos & Condutivo & Leve \\
\hline 5 & Feminino & 41 anos & Mista & Moderada \\
\hline 6 & Feminino & 28 anos & Mista & Moderada \\
\hline 7 & Feminino & 31 anos & Condutivo & Moderada \\
\hline 8 & Feminino & 35 anos & Mista & Moderada \\
\hline 9 & Feminino & 24 anos & Mista & Moderada/Severo \\
\hline 10 & Masculino & 39 anos & Mista & Moderada \\
\hline 11 & Feminino & 30 anos & Mista & Moderada \\
\hline 12 & Feminino & 37 anos & Mista & Moderada \\
\hline 13 & Feminino & 63 anos & Condutivo & Leve \\
\hline 14 & Feminino & 64 anos & Mista & Moderada/Severa \\
\hline 15 & Feminino & 33 anos & Mista & Moderada \\
\hline 16 & Feminino & 47 anos & Mista & Leve \\
\hline
\end{tabular}

Legenda: DA - deficiência auditiva

Figura 1 - Caracterização da amostra

A avaliação audiológica foi realizada em cabina acústica por meio do audiômetro da marca MA-41 Interacoustics calibrado segundo os padrões ANSI (1996). A audiometria tonal liminar foi realizada com os fones supra-aurais TDH-39 e fones de inserção ER-3A sendo os últimos adaptados ao meato acústico externo por meio de plugs descartáveis. Para a realização dos exames com os fones de inserção, foram utilizados os fatores de correção sugeridos pelo fabricante nas frequências de 250 a 8000 $\mathrm{Hz}$, aos quais foram acrescentados aos limiares obtidos ${ }^{1}$.

O teste de audiometria tonal liminar (ATL) por via aérea foi iniciado na frequência de $1000 \mathrm{~Hz}$, em 
seguida, foram testadas as frequências de $2000 \mathrm{~Hz}$, $3000 \mathrm{~Hz}, 4000 \mathrm{~Hz}, 6000 \mathrm{~Hz}, 8000 \mathrm{~Hz}, 500 \mathrm{~Hz}$ e $250 \mathrm{~Hz}$ nesta ordem pelo método descendente-ascendente ${ }^{13,14}$.

$\mathrm{Na}$ audiometria tonal liminar por via óssea, o vibrador ósseo foi posicionado sobre a mastóide, tendo sido pesquisados limiares tonais de $500 \mathrm{~Hz}$ a $400 \mathrm{~Hz}$. Assim como na ATL por via aérea, foi utilizado o método descendente-ascendente para a pesquisa do limiar ósseo ${ }^{13,14}$.

Para testar a via aérea com os fones supraaurais, o mascaramento foi utilizado sempre que houve diferença de $40 \mathrm{~dB}$ ou mais entre as orelhas por frequência e/ou quando o limiar da via óssea da orelha não-testada apresentou diferença de $40 \mathrm{~dB}$ ou mais em relação à via aérea da orelha testada ${ }^{12}$. Já para os fones de inserção o mascaramento foi utilizado sempre que houve diferença de $55 \mathrm{~dB}$ ou mais entre as orelhas por frequência e/ou quando o limiar da via óssea da orelha não-testada apresentou diferença de $55 \mathrm{~dB}$ ou mais em relação à via aérea da orelha testada ${ }^{15}$.

A utilização do mascaramento seguiu os passos: obteve-se os limiares de via aérea sem mascaramento, depois comparou-se com os limiares de via aérea e via óssea da orelha não testada, verificouse se a diferença era maior ou igual a $40 \mathrm{~dB}$ para o fone TDH-39 e/ou maior ou igual a $55 \mathrm{~dB}$ para o fone de inserção, estabeleceu-se o nível inicial de mascaramento para a orelha não testada, isto é, limiar de via aérea da orelha não testada acrescido de $10 \mathrm{~dB}$ de mascaramento efetivo (narrow band). Restabeleceu-se o limiar da orelha testada com este nível inicial de mascaramento, cada vez que o paciente respondeu ao sinal apresentado à orelha testada aumentou-se o mascaramento em $10 \mathrm{~dB}$ e cada vez que o paciente não respondeu ao sinal de tom puro apresentado à orelha testada aumentouse o tom puro em $5 \mathrm{~dB}$ até que ele respondesse novamente.O mascaramento foi considerado suficiente quando ao aumentar o ruído em dois intervalos de $10 \mathrm{~dB}$ não houve alteração do limiar na orelha testada ${ }^{12}$.

Já para a testagem da via óssea, o mascaramento foi sempre utilizado, pois considerou-se atenuação interaural igual a zero. O mascaramento por via óssea foi utilizado da seguinte maneira: a intensidade inicial do ruído foi de $10 \mathrm{~dB}$ a partir do limiar da via aérea da orelha não testada, obteve-se o limiar ósseo da orelha testada e caso a orelha não testada pudesse responder pela testada, o ruído foi aumentado em intervalos de $10 \mathrm{~dB}$ até que a resposta pudesse ser confirmada.

A suspeita de ocorrência do super-mascaramento foi levantada quando o aumento da quantidade de ruído mascarante provocou elevação proporcional do limiar da orelha testada em pelo menos duas oportunidades. No presente estudo, não foi possível verificar a ocorrência de super-mascaramento em nenhum dos pacientes avaliados, de modo que os limiares tanto por via aérea como óssea puderam ser confirmados sem a influência da orelha contralateral.

Para a realização dos exames com os fones de inserção, foram utilizados os fatores de correção sugeridos pelo fabricante nas frequências de $250 \mathrm{a}$ $8000 \mathrm{~Hz}$, já que o audiômetro utilizado no presente estudo não permite a correção automática dos limiares obtidos com os diferentes transdutores.

Os resultados obtidos na audiometria tonal, bem como as quantidades de mascaramento utilizadas com ambos os transdutores, foram comparados por meio de análise estatística considerando as variáveis: lado da orelha e tipo de transdutor.

O presente estudo teve seu projeto avaliado e aprovado pelo Comitê de Ética em Pesquisa da UNIFESP, sob o número 01532/07, tendo os indivíduos sido informados sobre os procedimentos a serem realizados e assinado o Termo de Consentimento Livre e Esclarecido para a participação do estudo e posterior divulgação dos resultados.

Para a comparação dos lados das orelhas (esquerda e direita), o mascaramento medido em nível de audição (dBNA) e o tipo de transdutor (TDH-39 e ER-3A) foi empregado o teste de MannWhitney, o qual é um teste paramétrico usado quando as amostras são independentes e desejase comparar sempre duas-a-duas as variáveis.

Estabeleceu-se para este estudo um nível se significância de $5 \%$ ( $p$ menor ou igual 0,05 ). Os resultados com significância estatística serão destacados com asterisco $\left(^{*}\right)$ e com tendência à significância serão destacados com o símbolo sustenido (\#). Os intervalos de confiança (IC) foram construídos com $95 \%$ de confiabilidade.

\section{RESULTADOS}

Após a aplicação do teste estatístico de MannWhitney observou-se que não houve diferença estatisticamente significante entre as orelhas direita e esquerda em todas as variáveis estudadas e com ambos os transdutores, sendo assim foi possível considerar ambas as orelhas ( $n=32$ orelhas) nas análises posteriores, o que conferiu ao estudo maior fidedignidade.

A tabela 1 compara a quantidade de mascaramento utilizada em dBNA entre os fones TDH-39 e os fones ER-3A para testar a via aérea, por frequência. 
Tabela 1 - Valores descritivos (média, mediana e desvio padrão) da quantidade de mascaramento utilizados com os fones TDH-39 e ER-3A em dBNA, para testar a via aérea (250Hz-8000Hz;SRT;IPRF) e logoaudiometria (SRT e IPRF)

\begin{tabular}{|c|c|c|c|c|c|c|c|}
\hline VA & Fones & Média & Mediana & Desvio Padrão & $\mathbf{N}$ & IC & p-valor \\
\hline \multirow{2}{*}{$250 \mathrm{~Hz}$} & TDH & 83,2 & 80 & 8,1 & 11 & 4,8 & \multirow{2}{*}{$0,027^{*}$} \\
\hline & ER-3A & 67,5 & 68 & 3,5 & 2 & 4,9 & \\
\hline \multirow{2}{*}{$500 \mathrm{~Hz}$} & TDH & 79,3 & 80 & 8,7 & 14 & 4,6 & \multirow{2}{*}{ 0,076\# } \\
\hline & ER-3A & 67,5 & 68 & 3,5 & 2 & 4,9 & \\
\hline \multirow{2}{*}{$1 \mathrm{kHz}$} & $\mathrm{TDH}$ & 77,2 & 75 & 13,9 & 9 & 9,1 & \multirow{2}{*}{$-x-$} \\
\hline & ER-3A & $-x-$ & $-x-$ & $-x-$ & 0 & $-x-$ & \\
\hline \multirow{2}{*}{$2 \mathrm{kHz}$} & $\mathrm{TDH}$ & 67,5 & 68 & 24,7 & 2 & 34,3 & \multirow{2}{*}{$-x-$} \\
\hline & ER-3A & $-x-$ & $-x-$ & $-x-$ & 0 & $-x-$ & \\
\hline \multirow{2}{*}{$3 \mathrm{kHz}$} & $\mathrm{TDH}$ & 57,5 & 55 & 12,6 & 4 & 12,3 & \multirow{2}{*}{0,147} \\
\hline & ER-3A & 100,0 & 100 & $-x-$ & 1 & $-x-$ & \\
\hline \multirow{2}{*}{$4 \mathrm{kHz}$} & $\overline{\mathrm{TDH}}$ & 68,8 & 70 & 11,6 & 8 & 8,0 & \multirow{2}{*}{0,423} \\
\hline & ER-3A & 60,0 & 60 & $-x-$ & 1 & $-x-$ & \\
\hline \multirow{2}{*}{$6 \mathrm{kHz}$} & TDH & 78,3 & 70 & 18,9 & 3 & 21,4 & \multirow{2}{*}{$-x-$} \\
\hline & ER-3A & $-x-$ & $-x-$ & $-x-$ & 0 & $-x-$ & \\
\hline \multirow{2}{*}{$8 \mathrm{kHz}$} & $\mathrm{TDH}$ & 81,7 & 80 & 17,6 & 3 & 19,9 & \multirow{2}{*}{1,000} \\
\hline & ER-3A & 80,0 & 80 & $-x-$ & 1 & $-x-$ & \\
\hline
\end{tabular}

Legenda: $p<0,05$ Wilcoxon. TDH: fone supra-aural TDH-39; ER-3A: fone de inserção ER-3A; Hz: hertz; Khz:quilohertz; N: número de indivíduos; IC: índice de confiança para média; VA: via aérea; -x-: não foi possível usar a estatística; dBNA:nível de audição;dBNS: nível de sensação.

A partir da observação da Tabela 1 verifica-se que as quantidades de mascaramento utilizadas com o fone de inserção ER-3A para testar a via aérea nas frequências de $250 \mathrm{~Hz}, 500 \mathrm{~Hz}, 4 \mathrm{KHz}, 8 \mathrm{KHz}$ foram menores do que as utilizadas com o fone supraaural TDH-39. Dentre estas, a frequência de $250 \mathrm{~Hz}$ por via aérea apresentou resultado estatisticamente significante e a frequência de $500 \mathrm{~Hz}$ apresentou tendência a significância estatística.

A tabela 2 compara a quantidade de mascaramento utilizada em dBNA entre os fones TDH-39 e os fones ER-3A para testar a via óssea, por frequência.

Tabela 2 - Valores descritivos (média, mediana e desvio padrão) da quantidade de mascaramento utilizadas com os fones TDH-39 e ER-3A em dBNA, para testar a via óssea nas frequências de $500 \mathrm{~Hz}-4000 \mathrm{~Hz}$

\begin{tabular}{cccccccc}
\hline VO & Fones & Média & Mediana & Desvio Padrão & N & IC & p-valor \\
\hline \multirow{2}{*}{$500 \mathrm{~Hz}$} & TDH & 71,9 & 73 & 17,2 & 24 & 6,9 & \multirow{2}{*}{0,336} \\
& ER-3A & 68,6 & 70 & 14,5 & 22 & 6,1 & \\
\hline \multirow{2}{*}{$1 \mathrm{kHz}$} & TDH & 65 & 70 & 17,9 & 25 & 7 & \multirow{2}{*}{0,527} \\
& ER-3A & 68,5 & 70 & 18,5 & 23 & 7,6 & \\
\hline \multirow{2}{*}{$2 \mathrm{kHz}$} & TDH & 60,5 & 60 & 14,5 & 22 & 6 & \multirow{2}{*}{0,813} \\
& ER-3A & 59,5 & 58 & 16 & 22 & 6,7 & \\
\hline \multirow{2}{*}{$3 \mathrm{kHz}$} & TDH & 65,4 & 60 & 14,1 & 26 & 5,4 & \multirow{2}{*}{$0,033^{*}$} \\
& ER-3A & 73 & 75 & 14,1 & 28 & 5,2 & \multirow{2}{*}{0,648} \\
\multirow{2}{*}{$4 \mathrm{kHz}$} & TDH & 70,5 & 70 & 15,4 & 20 & 6,7 & \multirow{2}{*}{} \\
& ER-3A & 68,6 & 68 & 15,7 & 22 & 6,6 & \\
\hline
\end{tabular}

Legenda: $p<0,05$ Wilcoxon .TDH: fone supra-aural TDH-39; ER-3A: fone se inserção ER-3A; Hz: hertz; Khz:quilohertz; N:número de indivíduos; IC: índice de confiança para média; VA:via aérea; VO: via óssea; -x-: não foi possível usar a estatística; dBNA:nível de audição;dBNS: nível de sensação 
Pelos resultados da Tabela 2, observou-se que a quantidade de mascaramento utilizada com o fone ER-3A foi maior do que com o fone supra-aural na frequência de $3 \mathrm{KHz}$ (alta frequência), com diferença estatisticamente significante.

\section{DISCUSSÃO}

O resultado da Tabela 1 pode ser explicado pelo fato da atenuação interaural fornecida pelos fones de inserção ser maior do que com o fone o fone supra-aural, pois há uma menor área do crânio exposta à vibração, o que contribui para a diminuição da intensidade de mascaramento necessária para testar a via aérea, evitando assim os dilemas do mascaramento em casos de gap bilateral ${ }^{4,15,16}$.

Este resultado é importante para evitar o super-mascaramento, sobretudo considerando a configuração audiométrica das deficiências auditivas condutivas e mistas que possuem gap bilateral, sendo o mesmo freqüentemente maior nas frequências baixas.

Outros estudos demonstraram que os fones de inserção ER-3A proporcionaram maior atenuação interaural do que os fones supra-aurais convencionais, em especial para as baixas frequências em indivíduos com perda auditiva de grau profundo unilateral e/ou em casos de anacusia unilateral ${ }^{5,10}$.

$\mathrm{Na}$ literatura especializada, encontramos alguns estudos que verificaram ainda que os fones de inserção ER-3A, promovem isolamento acústico significamente maior entre as duas orelhas nas frequências baixas em comparação com o fone supra-aural TH-49P, o que corrobora com os resultados deste estudo ${ }^{4}$.

Além disso, um estudo discutiu sobre os diferentes tipos de fones utilizados em audiologia clínica, cada qual com suas vantagens e limitações. Citaram que os fones supra-aurais apresentam pouca confiabilidade nas frequências baixas devido à variabilidade e a instabilidade do acoplamento entre o transdutor e a orelha, ocorrendo escape de ar entre o coxim do fone e o pavilhão auricular. Sendo assim, os indivíduos podem apresentar respostas por vibração com os fones supra-aurais nas frequências baixas, devido a grande área exposta à vibração, dificultando a um resultado mais fidedigno ${ }^{17}$.

$\mathrm{Na}$ Tabela 1 não foi possível aplicar testes estatísticos para algumas frequências com o transdutor ER-3A, pois não houve necessidade do uso de mascaramento devido ao aumento da atenuação interaural. Este dado confirmou uma das vantagens do uso do fone de inserção em patologias condutivas. Especificamente na frequência de $3000 \mathrm{~Hz}$ com o fone ER3A, a análise ficou prejudicada, pois somente um paciente necessitou de mascaramento contra oito nos quais não houve necessidade de utilização de mascaramento com o fone TDH.

Já na Tabela 2, na frequência de $3000 \mathrm{~Hz}$ o mascaramento necessário para testar a via óssea foi maior com o fone de inserção do que com o fone supra aural. Tal fato pode ser explicado pela limitação do fone de inserção para as altas frequências, por este motivo, deve-se utilizar os fatores de correção sugeridos pelo fabricante os quais devem ser subtraídos do limiar obtido com os fones de inserção em frequências altas em equipamentos calibrados para fones supra-aurais e que não prevêem a correção automática ao se selecionar o tipo de transdutor. Esta limitação também foi observada por outros autores ${ }^{1,15}$.

O presente estudo revelou dados importantes a respeito do uso dos fones de inserção na prática de audiologia clínica em casos de deficiência auditiva com gap bilateral.

Pode-se notar que os fones de inserção demonstraram necessitar de quantidade significamente menor de ruído mascarante nas baixas frequências $(250 \mathrm{~Hz}$ e $500 \mathrm{~Hz})$, ou seja, os resultados mostraram que os fones de inserção contribuíram para obtenção de limiares por via aérea mais precisos nas baixas frequências em indivíduos com deficiências auditivas condutiva e/ou mista bilateral, diminuindo a ocorrência e/ou o risco de super-mascaramento.

\section{CONCLUSÕES}

A partir da análise crítica dos resultados da quantidade de mascaramento utilizada com os fones supra-aurais e fones de inserção, realizados em indivíduos com deficiência auditiva condutiva e mista, conclui-se que há influência do tipo de transdutor na quantidade de mascaramento utilizado para obtenção dos limiares de via aérea e via óssea, principalmente nas frequências baixas. $\mathrm{Na}$ via aérea, nas frequências de $250 \mathrm{~Hz}, 500 \mathrm{~Hz}$, $1 \mathrm{KHz}, 2 \mathrm{KHz}, 4 \mathrm{KHz}, 8 \mathrm{KHz}$, verifica-se uma menor quantidade de mascaramento utilizado para o fone ER-3A.Na via óssea, nas frequências de $500 \mathrm{~Hz}$, $2 \mathrm{KHz}$ e $4 \mathrm{KHz}$ verifica-se uma menor quantidade de mascaramento utilizado para o fone ER-3A.

\section{AGRADECIMENTOS}

À Fundação de Amparo à Pesquisa do Estado de São Paulo (FAPESP) pelo apoio financeiro concedido por meio do projeto de iniciação científica $n^{\circ} 08 / 54682-7$. 


\section{ABSTRACT}

Purpose: this study aimed at examining the influence of transducer's type in the amount of masking needed to obtain air and bone conducted thresholds in subjects with conductive and mixed hearing losses. Method: 16 patients with symmetric bilateral conductive hearing deficiency or mixed hearing loss with air-bone gap of at least 15dBNA were examined. The subjects underwent clinical history, otoscopy and acoustic immittance measures (tympanometry and acoustic reflex), and subsequently, pure tone and speech audiometry (SRT and WRS). Pure tone threshold and speech audiometry were performed both with supra-aural TDH-39 and insertion earphones ER-3A. Results: there was no statistically significant difference between the ears regardless of the type of transducer. The amount of masking used with the insertion earphone ER-3A in order to test the air thresholds, in the frequencies of $250 \mathrm{~Hz}$ and $500 \mathrm{~Hz}$, was lower than the one used with supra-aural headset with statistical significance. Conclusions: transducer's type influences in the amount of masking used in order to obtain air and bone conducted thresholds, mainly in the low frequencies, whereas the amount of masking with the insertion phone is smaller, both for testing the air as well as the bone thresholds.

KEYWORDS: Hearing Loss, Conductive; Perceptual Masking; Hearing Aids; Audiometry, Pure-tone

\section{REFERÊNCIAS}

1. Gil D, Borges ACC. Fones de Inserção: Um estudo em indivíduos audiologicamente normais. Revista Brasileira Otorrinolaringologia. 2001;67(4):480-7.

2. Clemis JD, Ballad WJ, Killion MC. Clinical use of an insert earphone. The annals of Otology Rhinology and Laryngology.1986;95:520-4.

3. Lilly DJ, Purdy JK. On the routine use of tubephone insert earphones. Am. J. Audiol. 1993;2:17-20.

4. Sklare DA, Denenberg LJ. Interaural attenuation for tubephone insert earphones. Ear Hear. 1987;8 (5):298-300.

5. Van Campen LE, Sammeth CA, Peek BF. Interaural attenuation using ER-3A insert earphones in auditory brain stem response testing. Ear Hear. 1990;11(1):66-9.

6. Frank T, Wright DC. Attenuation provided by four different audiometric earphone systems. Ear Hear. 1990;11(1): 70-8.

7. Blackwell KL, Oyler RF, Seyfried DN. A clinical comparison of Grason Stadler insert earphones and TDH-50P standart earphones. Ear Hear.1990;12(5):361-2.

http://dx.doi.org/10.1590/S1516-18462011005000142

RECEBIDO EM: 15/10/2010

ACEITO EM: 13/06/2011

Endereço para correspondência:

Lívia Tamie Oshiro.

Rua Afonso Celso, 1425. Apto 34 - Vila Mariana

São Paulo - SP

CEP: 04119-062

E-mail: liviatamie@yahoo.com.br
8. Mueller HG, Hall JW .Pure tone audiometry. San Diego: Singular; 1997.Diagnostic Audiology, Principles, Procedures and Practices; p. 78-112.

9. Munro KJ, Agnew N. A comparison of inter-aural attenuation with the Etymotic ER-3A insert earphone and the Telephonics TDH-39 supra-aural earphone. Br.J.Audiol. 1999;33(4): 259-62.

10. Ramos JMP, Dabbur R, Gil D. Atenuação Interaural:Estudo Comparativo com dois tipos de transdutores. Rev Soc Bras de Fonoaudiol. 2009; 14(3):498-502.

11. Hosford-Dunn H, Kuklinski AL, Raggio M, Haggerty HS. Solving Audiometric masking dilemmas with an insert masker. Arch.Otolaryngol. Head Neck Surg.1986;112:92-5.

12. Katz J. Mascaramento Clínico Tomando decisões. Tratado de Audiologia Clínica. São Paulo: Manole;1999.p.109-31.

13. Munhoz MS, Caovilla HH, Silva MLG, Ganança MM. Audiologia Clínica. São Paulo:Atheneu;2000. Cap. 5. Audiometria tonal e vocal; p. 49-72.

14. Santos TMM, Russo ICP. A prática da audiologia clínica. São Paulo: Cortez; 2007. Determinação dos Limiares Tonais por Via aérea e por via óssea; p.67-95.

15. Killion MC, Wilber LA, Gudmendsen MA. Insert earphone for more interaural attenuation. Hearing Instruments.1985;36(2):34-6.

16. Zwislocki J. Acoustic attenuation between the ears. J. Acoust.Soc.Am.1953;25:752-9.

17. Zwislocki $\mathrm{J}$ et al. Earphones in audiometry. The Journal of the Acoustical Society of America.1988; 83(4):1688-9. 